\title{
NIVEL DE ACTIVIDAD FÍSICA EN ADOLESCENTES DE UN DISTRITO DE LA REGIÓN CALLAO
}

\author{
Juan Morales Quispe ${ }^{1, a}$, Roberto Jose Añez Ramos ${ }^{2, a}$, Cesar Abraham Suarez Oré $3, \mathrm{~b}$
}

\begin{abstract}
RESUMEN
Objetivos. Determinar el nivel de actividad física (AF) y la asociación con el uso excesivo de Internet entre los adolescentes del distrito de "Mi Perú", en la región Callao. Materiales y métodos. Estudio descriptivo transversal. Los sujetos participantes fueron estudiantes de 15 a 19 años de edad de los colegios estatales del distrito de "Mi Perú". Se empleó la versión larga del IPAQ (Physical Activity Questionnaire), fue aplicado en las aulas de clases entre octubre y diciembre del 2013. La AF se ha expresado en equivalente metabólico (MET) y se clasifico en nivel bajo, moderado y alto. El uso de Internet de más de $20 \mathrm{~h}$ semanales fue considerado excesivo. Resultados. Del total de participantes, el $59 \%$ (272) obtuvieron nivel alto de AF, el 25,8\% (119) nivel moderado de AF, y 15,2\% (70) nivel bajo de AF. En el $50 \%$ de los adolescentes el nivel de AF fue menor o igual a $4212 \mathrm{MET} / \mathrm{min} / \mathrm{sem}$. Según las áreas del IPAQ, se obtuvieron mayores niveles de AF en las áreas "labores de hogar" y "recreación". La AF en las labores del hogar fue mayor en el sexo femenino respecto a los varones $(p=0,010)$, mientras que en las actividades de recreación la AF fue mayor en los varones $(p=0,009)$. Solo el $2,6 \%(12)$ de los adolescentes usaron Internet más de $20 \mathrm{~h}$ semanales. Conclusiones. El estudio muestra un nivel alto de actividad física, no se encontró asociación entre el nivel de actividad física y el uso excesivo de Internet en la población estudiada.
\end{abstract}

Palabras clave: Actividad física; actividad motora; adolescente (Fuente: DeCS BIREME)

\section{LEVEL OF PHYSICAL ACTIVITY AMONG ADOLESCENTS IN A DISTRICT OF THE CALLAO REGION}

\begin{abstract}
Objectives. To determine the level of physical activity (PA) and the association of this factor with excessive Internet use among adolescents from the "Mi Perú" district in the Callao region. Materials and Methods. This cross-sectional descriptive study included 15-19-year-old students at state schools in the "Mi Perú" district. The long version of the Physical Activity Questionnaire (IPAQ) was applied in the subjects' classrooms between October and December 2013. PA was expressed in metabolic equivalent of task (MET) units and classified as low, moderate, and high. Internet usage of $\geq 20$ hours per week was considered excessive. Results. Of the total participants, 59\% (272), 25.8\% (119), and $15.2 \%$ (70) had high, moderate, and low levels of PA, and $50 \%$ of adolescents had a PA level $\leq 4,212 \mathrm{MET} / \mathrm{min} / \mathrm{wk}$. According to the IPAQ, higher levels of PA were achieved in the areas of "house work" and "recreation". The PA for house work was higher in female subjects relative to male subjects $(p=0.010)$, whereas the PA for recreation was higher among male subjects $(p=0.009)$. Only $2.6 \%(12)$ of teenagers used the Internet for $>20$ hours per week. Conclusions. This study reveals a high level of PA and a lack of association between the PA level and excessive Internet use in the studied population.
\end{abstract}

Key words: Physical activity; motor activity; adolescent (Fuente: MeSH NLM)

\section{INTRODUCCIÓN}

La Organización Mundial de la Salud (OMS) indica que la inactividad física constituye el cuarto factor de riesgo más importante de mortalidad en todo el mundo, después de la hipertensión, el consumo de tabaco y el exceso de glucosa en la sangre. La inactividad física, extendida en muchos países, tiene influencia en la prevalencia de las enfermedades no transmisibles (ENT) y en sus factores de riesgo ${ }^{(1)}$. El informe de la OMS sobre la situación mundial de las enfermedades no transmisibles, indica que de los 57 millones de muertes en el mundo ocurridos en 2008 , 36 millones $(63 \%)$ se debieron a las ENT y, de estos, el $80 \%$ ocurrieron en países de bajos y medianos ingresos. Entre los años 2010 y 2020, a nivel mundial se estima un incremento en un $15 \%$ de muertes por ENT ${ }^{(2)}$. Las personas con poca actividad física tienen entre el 20 y $30 \%$ más riesgo de mortalidad. La actividad física regular reduce el riesgo de enfermedad cardiovascular, incluyendo la hipertensión, diabetes, cáncer de mama y de colon, y la depresión (2).

\footnotetext{
Dirección Regional de Salud del Callao. Callao, Perú

Facultad de Medicina, Universidad del Zulia. Maracaibo, Venezuela.

Universidad de Ciencias y Humanidades. Lima, Perú.

Médico; ${ }^{\mathrm{b}}$ licenciado en Enfermería

Recibido: 14/12/2015 Aprobado: 04/05/2016
}

Citar como: Morales Quispe J, Añez Ramos RJ, Suarez Oré CA. Nivel de actividad física en adolescentes de un distrito de la región Callao. Rev Peru Med Exp Salud Publica. 2016;33(3):471-7. doi: 10.17843/rpmesp.2016.333.2312 
En el 2014, una publicación de la OMS, señalo que en Perú la mortalidad por enfermedades no transmisibles representa el $66 \%$ del total de las muertes ${ }^{(3)}$. Asimismo, la Encuesta Global de Salud Escolar reportó que menos de la cuarta parte de los estudiantes realizan alguna actividad física, mientras que más de la cuarta parte pasa de tres o más horas al día realizando actividades sedentarias como ver televisión, jugar en la computadora o navegar en Internet ${ }^{(4)}$.

Según las recomendaciones de la OMS, la práctica de actividad física moderada o vigorosa durante un mínimo de 60 min diarios ayuda a los niños y jóvenes a mantener un perfil de riesgo cardiorrespiratorio y metabólico saludable (1). El Colegio Americano de Medicina del Deporte (American College of Sports Medicine) y la Sociedad Americana del Corazón (American Heart Association) hacen referencia que la actividad física durante al menos 30 minutos (min) al día o realizar un mínimo de 150 min de actividad moderada a la semana, ha demostrado tener beneficios para la salud, mientras que perder o mantener el peso ideal, puede requerir al menos 30 min de actividad moderada casi todos los días de la semana ${ }^{(5)}$.

En Perú existen pocos estudios sobre el tema, teniendo en cuenta que los adolescentes de 10 a 19 años representan el $20,7 \%$ de la población, de los cuales, el $48,1 \%$ tienen edades comprendidas entre los 15 y 19 años ${ }^{(6)}$; por otro lado, en Perú durante el 2012 se perdieron 5800949 años de vida saludables (AVISA) por diversas enfermedades, siendo las no transmisibles $(60,5 \%)$ las más frecuentes; entre los 15 y 44 años de edad el $68,9 \%$ del total de AVISA fue debido a las enfermedades no transmisibles ${ }^{(7)}$. En el Callao, el grupo de las enfermedades no transmisibles representa el $64,5 \%$ del total de los AVISA ${ }^{(8)}$.

El objetivo del presente trabajo fue determinar el nivel de actividad física y la asociación con el uso excesivo de Internet entre los adolescentes estudiantes de las instituciones educativas públicas del distrito de "Mi Perú", en la región Callao.

\section{MATERIALES Y MÉTODOS}

Estudio descriptivo transversal. Se llevó a cabo en la jurisdicción del distrito de "Mi Perú", uno de los siete distritos que conforma la región Callao, en cuya jurisdicción existen numerosos asentamientos humanos con necesidades básicas insatisfechas. La población de estudio estuvo constituida por todos los adolescentes que cursaban el cuarto y quinto año de educación secundaria en los turnos de mañana y tarde de todas las instituciones educativas públicas (IEP) del distrito. De las cuatro IEP existentes, tres brindaron facilidades para el estudio, con una población total de 3225 estudiantes.
Los criterios de inclusión considerados fueron: ser estudiantes de una IEP y que al momento de la recolección de datos tuvieran una edad entre 15 a 19 años; la aplicación del instrumento se priorizó a los estudiantes que cursaban el cuarto y quinto año. La participación de los estudiantes fue voluntaria y se contó con el asentimiento y consentimiento informado. Se excluyeron a los adolescentes menores de 15 años que no completaron el llenado del cuestionario o que no aceptaron el asentimiento y/o el consentimiento informado. El principal motivo para no incluir adolescentes menores de 15 años fue la indicación del IPAQ (International Physical Activity Questionnaire), que señala su aplicación a partir de los 15 años.

Para tener una cifra de referencia del tamaño de la muestra se empleó la fórmula que considera la población finita, con un nivel de confianza del 95\%, proporción esperada del $5 \%$, precisión del $3 \%$ y una proporción esperada de pérdidas del $15 \%$, obteniéndose una muestra de 224 individuos. Por tratarse de una población concentrada y asequible, se consideró una muestra de 472 adolescentes. Las variables principales del estudio fueron la actividad física (AF), definida como todo movimiento del cuerpo que hace trabajar los músculos y que requiere más energía que estar en reposo ${ }^{(9)}$; y el uso excesivo de Internet.

\section{INSTRUMENTO DE MEDICIÓN}

Entre los instrumentos existentes para medir la actividad física está el cuestionario internacional de actividad física conocido como IPAQ por sus siglas en inglés, el cual es un instrumento aplicable a individuos de 15 a 65 años. Se decidió emplear dicho instrumento porque la población en el presente estudio está dentro del rango referido, además de ser el más usado en diversos países y recomendado por la OMS con propósitos de monitoreo e investigación. Los estudios de confiabilidad y validez del IPAQ se han hecho en doce países de seis continentes ${ }^{(10)}$ y se encuentra disponible en diferentes idiomas ${ }^{(11)}$

El instrumento empleado fue la versión larga del IPAQ que consta de 27 preguntas distribuidas en cinco áreas de actividad evaluados independientemente (1: actividad física relacionada con el trabajo; 2: actividad física relacionada con transporte; 3 : actividad física relacionada al trabajo de la casa, mantenimiento de la casa, y cuidado de la familia; 4: actividad física relacionada con la recreación, deporte y tiempo libre; y 5: actividad física relacionada con el tiempo dedicado a estar sentado). Las preguntas sobre el tiempo dedicado a estar sentado no se incluyen como parte de ningún resultado final de actividad física. El instrumento permite tener una valoración tanto continua como discreta del nivel de actividad física. 
La actividad física se ha expresado en equivalente metabólico (MET) y para su determinación se ha empleado los criterios considerados en la guía IPAQ ${ }^{(12)}$. Los resultados continuos se han expresado en METmin/sem: puntuación MET por minutos de actividad por días de la semana. Los valores que se emplearon para el análisis de los resultados fueron los establecidos por el IPAQ: andar al trabajo= 3,3 MET; bicicleta para ir al trabajo $=6,0 \mathrm{MET}$; trabajo en el patio moderada $=4,0$ $\mathrm{MET}$; intensidad vigorosa en ocio= 8,0 MET; con estos criterios se han obtenido los resultados por áreas y el resultado de la actividad física total que resulta de la suma de los resultados de los MET-min/sem de cada área. Los resultados discretos permiten clasificar en tres niveles de actividad física: bajo, moderado y alto.

El nivel "alto" se considera al cumplir con cualquiera de los siguientes criterios: actividades de intensidad vigorosa al menos 3 días sumando un mínimo total de actividad física de al menos $1500 \mathrm{MET}-\mathrm{min} / \mathrm{sem}$, o siete o más días de cualquier combinación de caminar, intensidad moderada o actividades de intensidad vigorosa sumando un mínimo total de actividad física de al menos 3000 MET-min/sem.

El nivel "moderado", si cumple con alguno de los siguientes criterios: tres días o más de actividad física vigorosa con una intensidad de al menos 20 min por día, o cinco o más días de intensidad física moderada y/o andar al menos 30 min por día, o cinco o más días de cualquier combinación de andar, actividad de intensidad moderada y actividad de intensidad vigorosa sumando un mínimo total de actividad física de al menos 600 MET-min/sem.

El nivel "bajo" incluye a los que no tienen actividad o presenta alguna actividad pero no lo suficiente para ser considerado en los niveles alto ni moderado.

La validez del instrumento se determinó mediante el juicio de expertos, con esta valoración se determinó el coeficiente $W$ de Kendall. Las magnitudes de concordancia obtenidos para cada ítems fue de $\mathrm{W}=0,625$ $(p=0,000)$, mientras que para la valoración global fue de $W=0,727(p=0,006)$, lo que indica la existencia de acuerdo entre los evaluadores. Las sugerencias de los evaluadores fueron tomadas en cuenta para adecuar el instrumento a nuestro entorno.

La fiabilidad del instrumento se determinó mediante una prueba piloto a 30 adolescentes con características similares a la muestra, se evaluó la recolección de información, la comprensión del cuestionario y el tiempo de aplicación del instrumento. Al determinar el coeficiente de confiabilidad, el alfa de Cronbach obtenido fue de 0,842. Para conocer el uso excesivo de
Internet, se midió su frecuencia y consumo, valorado en horas por semana, considerándose un uso excesivo cuando supera las $20 \mathrm{~h}$ semanales ${ }^{(13)}$.

\section{PROCEDIMIENTOS}

La técnica empleada para la recolección de información fue la encuesta y el instrumento que se usó fue el cuestionario. Previa autorización de los directores de las instituciones educativas y coordinaciones con los docentes, la aplicación del instrumento se realizó en las aulas de clases, entre octubre y diciembre de 2013 y estuvo bajo la supervisión de profesionales del área de salud, previamente capacitados, quienes estuvieron presentes durante todo el proceso que tomó la administración del instrumento. El equipo de encuestadores acudió hasta un máximo de tres veces a las aulas seleccionadas, en los turnos de mañana y tarde, asegurando así que toda la población de estudiantes, de cuarto y quinto año, tengan la misma probabilidad de ser incluidos en la muestra.

\section{ANÁLISIS ESTADÍSTICO}

En el análisis univariado se determinó las frecuencias y porcentajes según sexo, edad, tiempo de residencia, acceso a internet y la frecuencia de uso de Internet. En el análisis bivariado se utilizó la prueba de chi cuadrado para buscar asociación entre las características generales de la población y los niveles actividad física. Para el análisis no paramétrico se calculó la mediana (p25-p75), según las categorías del IPAQ, además se utilizó la prueba de $U$ de Mann-Whitney para encontrar diferencias según sexo. Se consideró un valor de $p<0,05$ como estadísticamente significativo. El análisis se realizó utilizando los programas Microsoft Excel 2010 y Statistical Package for the Social Sciences (SPSS) versión 20.0 .

\section{ASPECTOS ÉTICOS}

La participación de los estudiantes fue voluntaria, contando con el asentimiento y consentimiento informado firmado por los participantes y sus padres o apoderados respectivamente. El proyecto de investigación fue aprobado por el comité de ética de investigación de la Facultad de Medicina de la Universidad Nacional Mayor de San Marcos (Acta 0164).

\section{RESULTADOS}

De un total de 472 participantes, 11 fueron excluidos por no haber llenado la totalidad del cuestionario, quedando una muestra de 461 adolescentes, de los cuales 48,8\% (225) fueron de sexo masculino y $51,2 \%$ (236) de sexo femenino. El grupo mayoritario tenía 16 años $(47,5 \%)$, el $54,4 \%$ de los participantes han vivido más de 10 años en 
el distrito donde estudian. La mayoría de los participantes accedieron a Internet con una frecuencia de 2 a 3 veces por semana $(41,9 \%$ ) y el $76,1 \%$ consumieron Internet de 3 a 5 h por semana (Tabla 1 ).

En relación al nivel de AF en los adolescentes, el 59,0\% (272) de los participantes alcanzaron un nivel "alto", el $25,8 \%$ (119) el nivel "moderado", mientras que 15,2\% (70) un nivel "bajo". Según sexo, los varones 60,4\% (136) logra obtener nivel de AF alto. Según edad, la mayoría de los participantes tuvieron 16 años y fueron los que en mayor proporción obtuvieron un nivel alto de AF. Los participantes que residen en el distrito donde estudian obtienen en mayor proporción un nivel alto de AF. Los adolescentes que tienen acceso a Internet con una frecuencia de 2 a 3 veces/semana son los que en mayor proporción obtienen nivel alto de AF. Según el uso de internet en horas por semana, la mayoría consumen de 3 a $5 \mathrm{~h}$ por semana y, de este grupo, la mayor proporción

Tabla 1. Características generales de los adolescentes del distrito de "Mi Perú", región Callao, 2013

\begin{tabular}{|c|c|c|}
\hline Características & $\mathbf{n}$ & $\%$ \\
\hline \multicolumn{3}{|l|}{ Sexo } \\
\hline Masculino & 225 & 48,8 \\
\hline Femenino & 236 & 51,2 \\
\hline \multicolumn{3}{|l|}{ Edad (años) } \\
\hline 15 & 115 & 24,9 \\
\hline 16 & 219 & 47,5 \\
\hline 17 & 98 & 21,3 \\
\hline 18 & 24 & 5,2 \\
\hline 19 & 5 & 1,1 \\
\hline \multicolumn{3}{|l|}{ Tiempo de residencia * } \\
\hline Menos de 1 año & 44 & 9,5 \\
\hline $1-5$ años & 67 & 14,5 \\
\hline 5-10 años & 69 & 15,0 \\
\hline Mayor de 10 años & 251 & 54,4 \\
\hline No responde & 30 & 6,5 \\
\hline \multicolumn{3}{|l|}{ Acceso a Internet $†$} \\
\hline Todos los días & 151 & 32,8 \\
\hline De 2 a 3 veces/semana & 193 & 41,9 \\
\hline $1 \mathrm{vez} / \mathrm{semana}$ & 54 & 11,7 \\
\hline De 2 a 3 veces/mes & 16 & 3,5 \\
\hline Con menor frecuencia & 46 & 10,0 \\
\hline No responde & 1 & 0,2 \\
\hline \multicolumn{3}{|l|}{ Consumo de Internet $\ddagger$} \\
\hline De 3 a 5 h & 351 & 76,1 \\
\hline De 6 a $10 \mathrm{~h}$ & 43 & 9,3 \\
\hline De 11 a $15 \mathrm{~h}$ & 18 & 3,9 \\
\hline De 16 a $20 \mathrm{~h}$ & 9 & 2,0 \\
\hline Mayor a $20 \mathrm{~h}$ & 12 & 2,6 \\
\hline No responde & 28 & 6,1 \\
\hline Total & 461 & 100 \\
\hline
\end{tabular}

* Tiempo de residencia en el distrito donde estudian

† Frecuencia de acceso a Internet por semana

‡ Consumo de Internet en horas por semana obtiene nivel de AF alto; entre los que usan más de 20 $\mathrm{h}$ de Internet por semana, el 58,3\% (7) obtiene nivel de AF moderado. No se encontró asociación entre el nivel de AF y el sexo, grupo de edad, tiempo de residencia, frecuencia de acceso a Internet ni su consumo en horas por semana $(p>0,05)$ (Tabla 2).

Según las áreas del IPAQ, se obtuvieron mayores niveles de AF en las áreas "labores de hogar" (trabajo de la casa, mantenimiento de la casa y cuidado de la familia) y "recreación" (AF de recreación, deporte y tiempo libre). De manera global, en el $50 \%$ de los adolescentes el nivel de AF fue menor o igual a $4212 \mathrm{MET}-\mathrm{min} / \mathrm{sem}$; y al separar por áreas del IPAQ, los niveles de AF fueron notablemente inferiores. En el área del IPAQ correspondiente al "trabajo", los cuartiles Q1 (p25), Q2 (p50 o mediana) y Q3 (p75) coinciden y equivalen a cero, lo que indica que en por lo menos el $75 \%$ de los adolescentes el nivel de AF es cero MET-min/sem; la AF en el trabajo se observa desde el p95 con $2761 \mathrm{MET}-\mathrm{min} / \mathrm{sem}$, lo que quiere decir el $95 \%$ de la población estudiada no realiza AF en el trabajo. En el área del IPAQ correspondiente al transporte, el $50 \%$ de los adolescentes tienen un nivel de AF menor o igual a 462 MET-min/sem; en el área "labores de hogar", el $50 \%$ tiene un nivel de AF menor o igual a 1453 MET-min/ sem; mientras que en el área "recreación", el $50 \%$ de los adolescentes tienen un nivel de AF menor o igual a 855 MET-min/sem (Tabla 3).

Según el sexo, en los varones la AF en el trabajo comienza a observarse en el p90 con 480 MET-min/sem; y en las mujeres en el p95 con 2432,4 MET-min/sem. La AF en las tareas del hogar es mayor en el sexo femenino respecto a los varones $(p=0,010)$, mientras que en las actividades de recreación, la AF es mayor en los varones $(p=0,009)$. No hubo diferencias significativas entre sexos en el nivel de AF en el trabajo y en el transporte (Tabla 4).

\section{DISCUSIÓN}

En la población adolescente del distrito de "Mi Perú", existe un predominio del nivel alto de actividad física; entre las áreas del IPAQ que alcanzaron mayores niveles de actividad física estacan las "labores de hogar" y "recreación". Existe un predominio del sexo femenino en la AF correspondiente a las "labores de hogar", mientras que la AF en las actividades de "recreación" predomina en los varones. Las áreas del IPAQ donde se obtienen menores niveles de AF fueron el "trabajo" y "transporte", esto podría atribuirse a que los participantes al estar en la etapa escolar son dependientes de los padres, por lo que parte de la AF consiste en realizar los quehaceres del hogar y actividades de recreación en sus tiempos libres. Los resultados obtenidos por Dresch et al. en Brasil, son similares a los nuestros. En su estudio, el $57,5 \%$ (309) de los sujetos eran activos, de los cuales el 59,6\% (136) de los hombres y el $56 \%$ (176) de las mujeres se clasificaron como muy activos, sin embargo el nivel de 
Tabla 2. Características generales y nivel de actividad física de los adolescentes del distrito de "Mi Perú", región Callao, 2013

\begin{tabular}{|c|c|c|c|c|c|c|c|}
\hline \multirow{3}{*}{ Características } & \multicolumn{6}{|c|}{ Nivel de actividad física } & \multirow{3}{*}{$p^{*}$} \\
\hline & \multicolumn{2}{|c|}{ Alto } & \multicolumn{2}{|c|}{ Moderado } & \multicolumn{2}{|c|}{ Bajo } & \\
\hline & $\mathbf{n}$ & $\%$ & $n$ & $\%$ & $\mathbf{n}$ & $\%$ & \\
\hline Sexo & & & & & & & 0,087 \\
\hline Masculino & 136 & 60,4 & 49 & 21,8 & 40 & 17,8 & \\
\hline Femenino & 136 & 57,6 & 70 & 29,7 & 30 & 12,7 & \\
\hline Edad (años) & & & & & & & 0,431 \\
\hline 15 & 67 & 58,3 & 33 & 28,7 & 15 & 13,0 & \\
\hline 16 & 130 & 59,4 & 60 & 27,4 & 29 & 13,2 & \\
\hline 17 & 61 & 62,2 & 17 & 17,3 & 20 & 20,4 & \\
\hline 18 & 11 & 45,8 & 8 & 33,3 & 5 & 20,8 & \\
\hline 19 & 3 & 60,0 & 1 & 20,0 & 1 & 20,0 & \\
\hline Tiempo de residencia & & & & & & & 0,477 \\
\hline Menos de 1 año & 26 & 59,1 & 10 & 22,7 & 8 & 18,2 & \\
\hline 1-5 años & 36 & 53,7 & 21 & 31,3 & 10 & 14,9 & \\
\hline 5-10 años & 43 & 62,3 & 21 & 30,4 & 5 & 7,2 & \\
\hline Mayor de 10 años & 149 & 59,4 & 61 & 24,3 & 41 & 16,3 & \\
\hline Frecuencia de acceso a Internet & & & & & & & 0,485 \\
\hline Todos los días & 91 & 60,3 & 43 & 28,5 & 17 & 11,3 & \\
\hline De 2 a 3 veces/semana & 112 & 58,0 & 53 & 27,5 & 28 & 14,5 & \\
\hline $1 \mathrm{vez} / \mathrm{semana}$ & 32 & 59,3 & 10 & 18,5 & 12 & 22,2 & \\
\hline De 2 a 3 veces/mes & 11 & 68,8 & 3 & 18,8 & 2 & 12,5 & \\
\hline Con menor frecuencia & 26 & 56,5 & 10 & 21,7 & 10 & 21,7 & \\
\hline Consumo de Internet en horas por semana & & & & & & & 0,115 \\
\hline De 3 a $5 \mathrm{~h}$ & 209 & 59,5 & 90 & 25,6 & 52 & 14,8 & \\
\hline De 6 a $10 \mathrm{~h}$ & 32 & 74,4 & 6 & 14,0 & 5 & 11,6 & \\
\hline De 11 a $15 \mathrm{~h}$ & 10 & 55,6 & 5 & 27,8 & 3 & 16,7 & \\
\hline De 16 a $20 \mathrm{~h}$ & 6 & 66,7 & 1 & 11,1 & 2 & 22,2 & \\
\hline Mayor a $20 \mathrm{~h}$ & 5 & 41,7 & 7 & 58,3 & 0 & 0,0 & \\
\hline Total & 262 & 60,5 & 109 & 25,2 & 62 & 14,3 & \\
\hline
\end{tabular}

${ }^{*}$ Prueba de Chi cuadrado

AF fue estadísticamente superior en adolescentes del sexo masculino ${ }^{(14)}$.

Nuestros resultados discrepan de los hallazgos de Saucedo-Molina et al. en México, donde el 39,6\% de los adolescentes registró un nivel alto de AF. Además, encontraron diferencias significativas en sus tres modalidades (vigorosa, moderada y caminando), siendo los hombres quienes presentaron una media mayor de minutos por semana en comparación con las mujeres. Según sexo, se encontraron diferencias significativas $(p<0,01)$, siendo los hombres quienes realizan más AF alta (52,6\%) que las mujeres $(30,5 \%)$, mientras que las mujeres efectúan más $\mathrm{AF}$ baja $(39,3 \%)$ que los hombres $(21,3 \%)$. Los sujetos que según su índice de masa corporal fueron considerados de bajo peso y con desnutrición realizaban con mayor frecuencia un nivel alto de AF $(55,6 \%)$ en contraste con los que presentaron sobrepeso y obesidad $(38,7 \%)^{(15)}$. Cocca et al., en Granada, España, reportaron que los estudiantes de las escuelas de secundaria fueron más activos que los estudiantes universitarios, siendo los factores ambientales, psicológicos y sociales los que mostraron una influencia directa sobre la AF (16).

Estudios realizados en diversos contextos culturales han encontrado disminución de la AF con el progreso de la edad, entre la adolescencia a la adultez y según el sexo, mayor probabilidad de bajo nivel de AF en las mujeres, considerándose como hipótesis la maduración y desarrollo de las funciones reproductoras y menor estimulo hacia la AF por la familia y sociedad ${ }^{(17,18)}$.

Tabla 3. Comportamiento de la actividad física por áreas del IPAQ en los adolescentes del distrito de "Mi Perú", Región Callao, 2013

\begin{tabular}{ll}
\hline Áreas del IPAQ* & Mediana (p25-p75) \\
\hline Trabajo & $0(0-0)$ \\
Transporte & $462(0-990)$ \\
Hogar & $1453(360-3732)$ \\
Recreación & $855(0-2895)$ \\
Total & $4212(1866-8380)$ \\
\hline
\end{tabular}

* Medido en MET-min/sem, IPAQ: Physical Activity Questionnaire 
Tabla 4. Comportamiento de la actividad física por áreas del IPAQ según sexo en los adolescentes del distrito de "Mi Perú", región Callao, 2013

\begin{tabular}{lrrrr}
\hline \multirow{2}{*}{ Áreas del IPAQ* } & \multicolumn{1}{c}{ Masculino } & \multicolumn{1}{c}{ Femenino } & \multirow{2}{*}{$\boldsymbol{p}^{\dagger}$} \\
\cline { 2 - 3 } \cline { 5 - 6 } & Mediana (p25-p75) & Mediana (p25-p75) & 0,259 \\
Trabajo & $0(0-0)$ & $396(57,75-840,75)$ & 0,378 \\
Transporte & $462(0-1155)$ & & $1702(610,25-3873)$ & 0,010 \\
Hogar & $1095(99-3630)$ & & $536,25(49,50-2282,5)$ & 0,009 \\
Recreación & $1386(0-3615)$ & & $3928(1920-7721)$ & 0,441 \\
\hline Total & $4911(1548-8838)$ & & \\
\hline
\end{tabular}

* Medido en MET-min/sem † Prueba U de Mann-Whitney

IPAQ: Physical Activity Questionnaire

En nuestro estudio, el 15,2\% (70) obtienen un bajo nivel de $A F$, siendo el área de transporte donde se obtienen menores niveles de AF, lo que presumiblemente se deba a que los participantes, especialmente los del centro urbano, tienen fácil acceso a los vehículos motorizados de transporte público y para aquellos que poseen bicicletas, la infraestructura vial y seguridad es inadecuado e insuficiente. Bergmann et al. en Rio Grande do Sul, Brasil, encontró una prevalencia de inactividad física en los adolescentes de $68 \%$ y entre los principales factores asociados a la inactividad física destacaron el residir en apartamento, sexo femenino, percibirse menos activo que sus pares ${ }^{(19)}$. Similares resultados se encontraron en Fortaleza, donde aproximadamente el $68 \%$ de los jóvenes entre 12 y 17 años eran sedentarios. Este fue mayor en el sexo femenino $(p<0,001)$ y en los que tenían sobrepeso $(p=0,001)$. Entre los participantes activos el ejercicio más practicado fue el futbol (42\%) ${ }^{(20)}$.

En el distrito de "Mi Perú" existen muchos asentamientos humanos con necesidades básicas insatisfechas y en situación económica precaria, los centros de estudios generalmente se ubican a una considerable distancia, lo que resulta una oportunidad para realizar AF. Asare \& Danquah en Ghana, encontraron una alta conducta sedentaria en adolescentes africanos provenientes de un nivel económico alto, los cuales se asocian a una mala salud mental (21). En São Paulo, entre los adolescentes que estudian en el turno noche la prevalencia global de la actividad física es baja y significativamente mayor en individuos más jóvenes y en adolescentes con menor nivel socioeconómico ${ }^{(22)}$.

No se encontró asociación entre el nivel de actividad física y el consumo excesivo de Internet, lo que podría deberse a que tanto, el uso de Internet como la actividad física se dan en diferentes momentos. Además, el servicio de Internet es todavía limitado en la jurisdicción donde se llevó a cabo el estudio. Martí, encontró que estar frente a diferentes pantallas por más de $4 \mathrm{~h}$ diarias se asoció a un aumento en $2 / 3$ del riesgo de fallar en el nivel adecuado de AF (23). Prieto-Benavides et al., en Bogotá, encontraron que los participantes que permanecieron menos de dos horas al día frente a TV/TV por cable, computador/Internet y/o consolas de videojuegos, completaron tiempos mayores a $60 \mathrm{~min}$ por día de actividad física ${ }^{(24)}$.
Como limitaciones, consideramos que la aplicación del cuestionario, en horas de clases, sumado a la extensión del instrumento, pudo haber condicionado a culminar con mayor prontitud el llenado del instrumento. Asimismo, el hecho de emplear un cuestionario como instrumento de medición ${ }^{(25)}$ puede no recoger AF de la vida diaria no planificada, ya que el autoinforme puede subestimar o sobreestimar la AF, que a diferencia de los dispositivos electrónicos como los podómetros y acelerómetros cuantifican objetivamente la AF, pero tampoco están libres de limitaciones, entre ellas el costo ${ }^{(26)}$. La literatura señala que los módulos de actividades domésticas y laborales de la versión larga del IPAQ generan confusión y sobreestiman la actividad física (27); sin embargo, en el distrito de "Mi Perú", donde existen hogares con necesidades básicas insatisfechas, mientras los padres trabajan fuera de la jurisdicción del distrito, los hijos cooperan con las labores del hogar, realizando AF.

En conclusión, el presente estudio muestra un nivel alto de actividad física en adolescentes, sobre todo en las áreas de labores de "hogar" y "recreación" con diferencias significativas entre sexos, no se ha encontrado asociación entre el nivel de actividad física y el uso excesivo de Internet entre los adolescentes del distrito de "Mi Perú".

Contribuciones de autoría: JMQ, participo en la concepción y diseño de la investigación, recolección y obtención de resultados, análisis e interpretación de datos y en la redacción del artículo; RJAR, participó en la asesoría estadística, revisión crítica del artículo y aprobación de la versión final; CASO participó en el análisis e interpretación de datos, aporte de materiales de estudio, asesoría administrativa y aprobación de la versión final del artículo.

Agradecimientos: a todos los directores y docentes de las instituciones educativas públicas del distrito de "Mi Perú" de la región Callao, por haber dado las facilidades para la aplicación del instrumento de recolección de datos, especialmente a Francisco Flores Jamanca, Víctor Benigno Cárdenas Peña, Luz Ríos Cuadros y Clara Velasco de Leyva. AAna María Guerrero Rodas, enfermera del centro de salud "Mi Perú" y a sus colaboradores, por haber participado en la aplicación del instrumento de recolección de datos. A los docentes de la Universidad de Ciencias y Humanidades, por sus sugerencias y recomendaciones.

Fuentes de financiamiento: autofinanciado

Conflictos de interés: declaramos no tener conflictos de interés. 


\section{REFERENCIAS BIBLIOGRÁFICAS}

1. Organizacion Mundial de la Salud. Recomendaciones Mundiales Sobre Actividad Fisica Para la Salud. Ginebra: OMS; 2010.

2. World Health Organization. Global status report on noncommunicable diseases 2010. Geneva: WHO; 2011.

3. World Health Organization. Noncommunicable Diseases. Country Profiles 2014. Geneva: WHO; 2014.

4. Perú, Ministerio de Salud. Encuesta Global de Salud Escolar ResultadosPeru 2010. Lima: MINSA; 2011.

5. American College of Sports Medicine. ACSM, AHA Support Federal Physical Activity Guidelines [Internet]. Acceso 10/10/2015. Indianapolis: ACSM; 2011 [cited 2016 Jan 27]. Disponible en: https://www.acsm.org/ about-acsm/media-room/acsm-in-thenews/2011/08/01/acsm-aha-supportfederal-physical-activity-guidelines

6. Instituto Nacional de Estadistica e Informatica. Censos Nacionales 2007. XI de población y VI de vivienda [Internet]. Lima: INEI; 2007 [cited 2016 Jan 27]. Disponible en: http://censos.inei.gob.pe/cpv2007/ tabulados/default.asp\#

7. Perú, Ministerio de Salud. Carga de Enfermedad en el Peru. Estimación de los años de vida saludables perdidos 2012. Lima: MINSA; 2014.

8. Perú, Ministerio de Salud, Dirección General de Epidemiología. Carga de Enfermedad de la Provincia de Lima y la Region Callao-2010. Lima: MINSA/ DGE; 2011.

9. Nacional Heart, Lung and BI. ¿Qué es la actividad física? [Internet]. Bethesda (MD): NIH; 2012 [updated 2012 Dec 28; cited 2016 Jan 27]. Disponible en: http://www.nhlbi.nih.gov/healthspanish/health-topics/temas/phys

10. Matsudo S, Araujo T, Matsudo V, Andrade D, Andrade E, Oliveira L, et al. Questionario Internacional de Atividade Fisica (IPAQ): Estudo de Validade e Reprodutibilidade No Brasil. Rev Bras Atividade Fis Saude. 2001;6(2):05-18.

11. International Physical Activity Questionnaire. Acceso 10.10.2015 [Internet]. IPAQ; 2010 [cited 2016 Jan 27]. Disponible en: www.sites.google. $\mathrm{com} /$ site/theipaq/questionnaire_links

12. Universidad de Granada. Junta de Andalucia. Traducción de las Guías para el Procesamiento de Datos y Análisis del Cuestionario Internacional de Actividad física (IPAQ) Versiones Corta y Larga [Internet]. Andalucia: UGR; 2005. Disponible en: http:// http://www.juntadeandalucia.es/salud/ sites/csalud/galerias/documentos/ p_4_p_2_promocion_de_la_salud/ actividad_fisica_alimentacion equilibrada/IPAQ_Guia_Traducida.pdf

13. Viñas F. Uso autoinformado de Internet en adolescentes: Perfil psicológico de un uso elevado de la red. Int J Psychol Psychol Ther. 2009;9(1):109-22.

14. Dresch F, Bertoli J, Silva J, Frutuoso A, Freitas C. Physical Activity Level of Students of a Public School in a City of Rio Grande do Sul. Rev Bras Promoc Saude, Fortaleza. 2013;26(3):360-5.

15. Saucedo-Molina Tde J, Rodríguez Jiménez J, Oliva Macías LA, Villarreal Castillo M, León Hernández RC, Fernández Cortés TL. Relacion entre el indice de masa corporal, la actividad fisica y los tiempos de comida en adolescentes mexicanos. Nutr Hosp. 2015;32(3):1082-90. doi: 10.3305/ nh.2015.32.3.9331.

16. Cocca A, Viciana-Ramírez J, MayorgaVega D, Ceballos-Gurrola O, Liukkonen J, Cocca M. Adolescents' and young adults ' physical activity related to built environment. Educ Fis y Cienc [Internet]. 2015 junio [cited 2016 Jan 27]; 17(1): [10 pág.]. Disponible en: http://www.scielo.org.ar/pdf/efyc/ v17n1/v17n1a04.pdf

17. Wasilewska M, Bergier J. Physical Activity Level of the Youth in Selected Countries of the World. Heal Probl Civiliz. 2015;9(3):39-46.

18. Dumith SC, Gigante DP, Domingues MR, Kohl HW 3rd. Physical activity change during adolescence: a systematic review and a pooled analysis. Int J Epidemiol. 2011;40:685-98. doi: 10.1093/ije/dyq272.

19. Bergmann GG, Bergmann ML, Marques AC, Hallal PC. Prevalence of physical inactivity and associated factors among adolescents from public schools in Uruguaiana, Rio Grande do Sul State, Brazil. Cad Saude Publica. 2013 Nov;29(11):2217-29.

20. De Freitas RW, da Silva AR, de Araújo MF, Marinho NB, Damasceno MM, de Oliveira MR. Prática de atividade física por adolescentes de Fortaleza, CE, Brasil. Rev Bras Enferm. 2010;63(3):410-5.

21. Asare M, Danquah SA. The relationship between physical activity, sedentary behaviour and mental health in Ghanaian adolescents. Child Adolesc Psychiatry Ment Health. 2015;9:11. doi: 10.1186/s13034-015-0043-x.

22. Ceschini FL, Andrade EL, Junior A. Physical activity and associated factors amongstudentsattendingeveningclasses. Rev Bras Cineantropom Desempenho Hum. 2015;17(2):205-15. doi: http://dx.doi.org/10.5007/19800037.2015v17n2p205.

23. Martí S. Actividad física, sedentarismo frente a pantallas y su relación en adolescentes [tesis doctorado]. Canarias: Universidad de las Palmas de Gran Canarias; 2011.

24. Prieto-Benavides DH, Correa-Bautista JE, Ramirez-Velez R. Niveles de actividad física, condición física y tiempo en pantallas en escolares de Bogotá, Colombia: Estudio FUPRECOL. Nutr Hosp. 2015;32(5):2184-92. doi: 10.3305/nh.2015.32.5.9576.

25. Ara I, Aparicio-Ugarriza R, MoralesBarco D, Nascimento de Souza W, Mata E, González-Gross M. Physical activity assessment in the general population; validated self-report methods. Nutr Hosp. 2015;31 Suppl 3:211-8. doi: 10.3305/nh.2015.31.sup3.8768.

26. Aparicio-Ugarriza $R$, Aznar $S$, Mielgoayuso J, Benito PJ, Pedrerochamizo R, Ara I, et al. Estimación de la actividad física en población general : métodos instrumentales y nuevas tecnologías. Rev Esp Nutr Comunitaria. 2015;21(Supl.1):215-24. DOI: 10.14642/RENC.2015.21.sup1.5068.

27. Hallal PC, Gómez LF, Parra DC, Lobelo F, MosqueraJ,Florindo A, et al.Lecciones aprendidas después de 10 Años del uso de IPAQ en Brasil y Colombia. J Phys Act Heal. 2010;7(Suppl 2):S259-64.

Correspondencia: Juan Morales Quispe

Dirección: Av. Universitaria 5175, Los Olivos. Lima, Perú

Teléfono: (+511) 989521832

Correo electrónico:mdjuanmorales@gmail.com 